\title{
Behavior Disorder and Social Function Impairment in Children with Basal Ganglia Germ Cell Tumors
}

This article was published in the following Dove Press journal:

Neuropsychiatric Disease and Treatment

\author{
Wenyi Lv' \\ Jin Feng ${ }^{\prime}$ \\ Li Chen' \\ Shuai Liu ${ }^{1,2}$ \\ Xiaoguang Qiu (iD) ${ }^{1,2}$ \\ 'Department of Radiation Oncology, \\ Beijing Tiantan Hospital, Capital Medical \\ University, Beijing 100070, People's \\ Republic of China; ${ }^{2}$ Beijing Neurosurgical \\ Institute, Capital Medical University, \\ Beijing 100070, People's Republic of \\ China
}

Purpose: Basal ganglia intracranial germ cell tumors (iGCTs) can specifically destroy the basal ganglia network, leading to several cognitive, learning, behavioral, and social impairments. This study aimed to investigate the behavior and social disorders of patients with basal ganglia iGCTs. Patients and Methods: We recruited 30 newly diagnosed iGCTs patients (and their parents) for the current study. The Child Behavior Checklist/6-18 was used to evaluate emotional and behavioral problems. The Conner's Parent Rating Scales was used to assess symptoms of hyperactivity/impulsivity and conduct problems. The health-related quality of life (HRQoL) was assessed using the Pediatric Quality of Life Inventory 4.0 Generic Core Scale. Performance status was assessed using the Lansky play-performance scale and Karnofsky performance scale. The effects of basal ganglia lesions on these scores were examined.

Results: Patients with basal ganglia iGCTs $(n=10)$ had more behavioral problems (attention problems, aggressive behavior, learning problems, hyperactivity index), social function impairment, anxiety/depression, and poorer HRQoL compared to patients with non-basal ganglia iGCTs $(n=20)$. There was no significant difference in the Lansky play-performance /Karnofsky performance scale scores.

Conclusion: This study demonstrates the effects of basal ganglia lesions on behavioral and emotional outcomes, social functions, and HRQoL of patients with iGCTs. The results may help to understand the function of basal ganglia and provide evidence for the benefit of early psychological intervention to improve the treatment for this rare disease.

Keywords: intracranial germ cell tumors, pediatrics, The Child Behaviour Checklist, The Conner's Parent Rating Scales, health-related quality of life

\section{Plain Language Summary}

Patients with intracranial germ cell tumors (iGCTs) have high changes of surviving the tumor, which leads to rising concerns over neuropsychological outcomes. Basal ganglia iGCTs account for $5-10 \%$ of all iGCTs cases. In clinical practice, parents of patients with basal ganglia iGCTs often report poor academic performance, attention deficiency, and interpersonal problems. We compared behavioral and emotional outcomes, social functions, and health-related quality of life based on tumor location in 30 newly diagnosed patients (and their parents) with iGCTs.

This study revealed that patients with basal ganglia iGCTs had more behavioral problems (attention problems, aggressive behavior, learning problems, hyperactivity index), greater social function impairment, a higher degree of anxiety/depression, and poorer health-related quality of life than patients with non-basal ganglia iGCTs. The results will improve our understanding of basal ganglia function and helps to provide new hypotheses for studying this rare disease. Effective psychosocial support interventions should be considered. 


\section{Introduction}

Patients with intracranial germ cell tumors (iGCTs) have high changes of surviving the tumor, which leads to rising concerns over neuropsychological outcomes. iGCTs are most common in the midline area, mainly in the pineal and suprasellar regions. ${ }^{1}$ They can also occur in the basal ganglia or thalamus or spread along the brain ventricles. Basal ganglia iGCTs account for 5-10\% of all iGCTs cases and have a higher incidence in Asian populations than European populations, with a male predominance., ${ }^{2,3}$

In addition to the well-known role of the basal ganglia in motor control, they play an important role in determining complex cognitive behavioral patterns and influencing the development of a child's brain. Specific destruction of the basal ganglia network directly leads to several primary cognitive, learning, memory, language, and behavioral disorders. $^{4-6}$ Disturbances of cortico-basal gangliathalamocortical circuits are related to several psychiatric and neurological disorders, such as Parkinson's disease, Tourette syndrome, attention deficit hyperactivity disorder (ADHD), addiction, and schizophrenia. ${ }^{7}$ Patients with basal ganglia iGCTs have progressive hemiparesis of the face and limbs and poorer neurocognitive function than patients with pineal or suprasellar lesions. ${ }^{8}$ Unfortunately, very few studies have investigated behavioral and social disorders in patients with basal ganglia iGCTs.

To fill these gaps, we compared behavioral and emotional outcomes, social functions, and the health-related quality of life (HRQoL) according to the tumor location, to depict the specific characteristics of basal ganglia iGCTs.

\section{Patients and Methods}

\section{Participants}

We recruited 33 patients treated for iGCTs at the Beijing Tiantan Hospital between August 2019 and January 2020, along with their parents. Thirty (91\%) families agreed to participate. Inclusion criteria were (1) age between 6 and 17 years, (2) new diagnosis of an iGCTs without other brain diseases, and (3) assessment before radiation treatment. Patients were diagnosed based on raised serum or cerebrospinal fluid tumor markers and consistent radiological appearances, or the biopsy and surgical resection. Before the evaluation (Table 1), five patients with pineal tumors underwent endoscopic third ventriculostomy (ETV) or ventriculoperitoneal shunt surgery (V-P shunt) to reduce hydrocephalus, while three patients received two or three cycles of chemotherapy.
This study was conducted in accordance with the Declaration of Helsinki and approved by the Ethics Committee of Beijing Tiantan Hospital. Patients with newly diagnosed iGCTs were referred to a neuro-oncologist after providing informed consent to evaluate their neuropsychological outcomes. All pediatric patients completed the child self-report (CSR) and the parent who spent the most time with the child was asked to complete the parent proxy report (PPR). A research assistant was available to read the questions to illiterate or visually impaired children. Assessments were conducted in Chinese and administered in person.

\section{Assessment Tools}

The Child Behavior Checklist (CBCL)/6-18 was used to measure the emotional and behavioral problems that had strong reliability and validity. ${ }^{9}$ The $\mathrm{CBCL} / 6-18$ has 113 items with ratings of behavioral, emotional, and social problems. The total problem score is the sum of scores for eight syndromes (anxious/depression, withdrawal/ depression, somatic complaints, social problems, thought problems, attention problems, rule-breaking behavior, and aggressive behavior), with a higher score indicating more behavioral problems. Parents match each item based on their child's current behavior and the behavior in the past six months. According to the guidelines, statistical analysis should use raw scale scores rather than $\mathrm{T}$ scores in order to take into account the full range of variation. ${ }^{9}$ The borderline clinical range behavioral problems spanned from the 93rd to the 97th percentile of the American norm, whereas above the 97th percentile reflected clinical-range behavioral problems. The total problem raw score of the common Chinese population was 21.61 (standard deviation (SD) = 19.978). The 90th and 95th percentiles for the total problem T score were 48.00 and 59.00, respectively. ${ }^{10}$

Parents also completed the Conners' Parent Rating Scales (CPRS), which assesses the symptoms of hyperactivity/impulsivity and conduct problems. ${ }^{11}$ Parents were asked to select from four possible responses based on the child's behavior, with a higher score indicating greater symptomatology. To obtain as much data as possible, we used the version of CPRS that has 48 questions, with a four-level scoring method $(0,1,2,3)$. The subscale includes conduct problem, learning problems, psychosomatic problems, impulsive-hyperactive behavior, anxiety, and hyperactivity index. ${ }^{12} \mathrm{~A}$ factor score higher than 1.5 indicates an abnormality (or a deviation of two standard deviations). 
Table I Demographics and Clinical Characteristics

\begin{tabular}{|c|c|c|c|}
\hline Characteristics & Basal Ganglia Group (\%) & Non-Basal Ganglia Group (\%) & $P$-value \\
\hline Number & 10 & 20 & \\
\hline Age, y (median, range) & $10.6,7.2-15.5$ & $12.0,6.3-16.8$ & $0.248^{\mathrm{a}}$ \\
\hline Male & $9(90)$ & $13(65)$ & $0.210^{\mathrm{b}}$ \\
\hline No. of lesions & & & $0.372^{\mathrm{b}}$ \\
\hline Single & $7(70)$ & $17(85)$ & \\
\hline Multiple & $3(30)$ & $3(15)$ & \\
\hline \multicolumn{4}{|l|}{ Location } \\
\hline Basal ganglia & $7(70)$ & 0 & \\
\hline Basal ganglia + Suprasellar & $2(20)$ & 0 & \\
\hline Basal ganglia + Suprasellar + pineal & $\mathrm{I}(\mathrm{I0})$ & 0 & \\
\hline Suprasellar & 0 & $10(50)$ & \\
\hline Pineal & 0 & $7(35)$ & \\
\hline Suprasellar + Pineal & 0 & $3(15)$ & \\
\hline Diagnosis & & & $1.000^{\mathrm{b}}$ \\
\hline Diagnosed by tumour markers & $7(70)$ & $13(65)$ & \\
\hline Diagnosed by pathology & $3(30)$ & $7(35)$ & \\
\hline \multicolumn{4}{|l|}{ Surgery type ${ }^{c}$} \\
\hline No surgery & $7(70)$ & $10(50)$ & $0.440^{\mathrm{b}}$ \\
\hline Definitive surgery & $\mathrm{I}(10)$ & $2(10)$ & $1.000^{\mathrm{b}}$ \\
\hline Biopsy only & $2(20)$ & $3(15)$ & \\
\hline ETV & 0 & I (5) & \\
\hline ETV + Biopsy & 0 & $2(10)$ & \\
\hline V-P shunt & 0 & $2(10)$ & \\
\hline \multicolumn{4}{|l|}{ Chemotherapy $^{d}$} \\
\hline Yes & $3(30)$ & 0 & \\
\hline No & $7(70)$ & $20(100)$ & \\
\hline
\end{tabular}

Notes: ${ }^{a}$ mann-Whitney $U$-test; ${ }^{\mathrm{b}}$ Fisher's exact test; ${ }^{\mathrm{c}}$ The median time between surgery and assessment was 24 days, range: $4-336$ days; ${ }^{\mathrm{d}}$ The patients received two or three cycles of chemotherapy before assessment.

Abbreviations: ETV, endoscopic third ventriculostomy; V-P shunt, ventriculoperitoneal shunt.

The Pediatric Quality of Life Inventory (PedsQL) 4.0 Generic Core Scale was used to assess HRQoL. ${ }^{13}$ The PedsQL generic core scale is widely used for both healthy populations and populations with acute or chronic health conditions. Physical, emotional, social, and school functioning are measured and reported as sub-scores. This scale also calculates the total scale score (TSS), physical health summary score (PHSS), and psychosocial health summary score (PsHSS). We used age-appropriate versions. All PedsQL scores are scaled from 0 to 100 , with higher scores indicating better HRQoL. ${ }^{14}$

Performance status was assessed using the Lansky play-performance scale (LPS) for children aged 6 to 15 years and the Karnofsky Performance Scale (KPS) for children aged over 16 years. ${ }^{15}$ These scales are commonly used to assess the functional status of patients with brain tumors. ${ }^{16,17}$

\section{Statistical Analyses}

Data were evaluated using IBM SPSS Statistics 25 and by analyzing descriptive statistics (frequency, number $(\mathrm{N})$, mean (M), SD). Fisher's exact test was used to compare categorical variables. The Mann-Whitney $U$-test was used to compare the behavioral and emotional outcomes, HRQoL, and performance status between patients with basal ganglia iGCTs and non-basal ganglia iGCTs. The Benjamini-Hochberg procedure was performed to control the false discovery rate (FDR). An adjusted p-value (q) < 0.1 was considered significant.

\section{Results}

Table 1 lists the participants' characteristics. Overall, $73.3 \%$ of the patients were male and the mean age at the time of diagnosis was 11.5 years (range: $6.3-16.8$ years). Ten of the 30 patients had basal ganglia lesions. Of the 
lesions in these ten patients, seven lesions invaded only the basal ganglia, while three invaded both basal ganglia and other brain regions. For all patients, the LPS/KPS was 74 $(\mathrm{SD}=16.10)$, HRQoL PPR TSS was $77.64(\mathrm{SD}=12.63)$, HRQoL CSR TSS was 78.37 (SD = 13.21), CPRS hyperactivity index was $0.34(\mathrm{SD}=0.31)$, and $\mathrm{CBCL}$ total score was $31.1(\mathrm{SD}=18.07)$.

Table 2 presents the comparison of all outcomes between basal ganglia lesions and non-basal ganglia lesions. Patients with basal ganglia lesions had higher scores on most children behavioral and emotional tasks than those with non-basal ganglia lesions in the CBCL (Figure 1, Table 2, anxiety/depression, social problems, attention problems, aggressive behavior, and total problems) and CPRS (learning problem and hyperactivity index). Compared to patients with non-basal ganglia lesions, patients with basal ganglia lesions had more social problems based on the social functioning subscores of both PPR and CSR in PedsQL and CBCL. The HRQoL of patients with basal ganglia lesions was worse than that of patients with non-basal ganglia lesions based on TSS and PsHSS of both PPR and CSR in PedsQL. There was no significant difference in the LPS/KPS score.

Based on the standard baseline of the common Chinese population, the CBCL total problems $\mathrm{T}$ score of the basal ganglia group was $60.01(\mathrm{SD}=5.08)$ and that of the non-basal ganglia group was $52.12(\mathrm{SD}=$ 9.53, $\mathrm{p}=0.006)$. All patients with basal ganglia lesions scored higher than the 90th percentile (48.00), and 50\% scored higher than the 95th percentile (59.00). Fifty percent of patients with non-basal ganglia lesions scored higher than the 90th percentile, and 10\% scored higher than the 95th percentile.

\section{Discussion}

This study examined behavioral and emotional outcomes, social functions, and HRQoL of patients with newly diagnosed iGCTs. Patients with basal ganglia lesions had more behavioral problems (attention problems, aggressive behavior, learning problems, higher hyperactivity index), greater social function impairment, higher levels of anxiety/depression, and poorer HRQoL compared to patients with non-basal ganglia lesions.

In clinical practice, parents of patients with basal ganglia iGCTs often report poor academic performance, attention deficiency, and interpersonal problems. As expected,
Table 2 Overall Outcomes of the Tumour Location Analysis

\begin{tabular}{|c|c|c|c|}
\hline & $\begin{array}{l}\text { Basal } \\
\text { Ganglia } \\
\text { M (SD) }\end{array}$ & $\begin{array}{l}\text { Non-Basal } \\
\text { Ganglia } \\
\text { M (SD) }\end{array}$ & q-value \\
\hline $\begin{array}{l}\mathbf{N} \\
\text { LPS/KPS }\end{array}$ & $\begin{array}{l}10 \\
80.00(11.55)\end{array}$ & $\begin{array}{l}20 \\
71.00(17.44)\end{array}$ & 0.287 \\
\hline $\begin{array}{l}\text { PedsQL-PPR } \\
\text { TSS } \\
\text { PHSS } \\
\text { PsHSS } \\
\text { Emotional } \\
\text { Social } \\
\text { School }\end{array}$ & $\begin{array}{l}70.98(7.43) \\
75.31(10.46) \\
68.67(7.49) \\
73.50(20.82) \\
72.50(16.37) \\
60.00(9.43)\end{array}$ & $\begin{array}{l}80.98 \text { (I3.50) } \\
80.31 \text { (I8.20) } \\
81.33 \text { (I3.30) } \\
82.25 \text { (15.00) } \\
88.75 \text { (I2.55) } \\
73.00 \text { (I8.45) }\end{array}$ & $\begin{array}{l}\mathbf{0 . 0 3 6} \\
0.305 \\
\mathbf{0 . 0 2 9} \\
0.423 \\
\mathbf{0 . 0 2 9} \\
0.143\end{array}$ \\
\hline $\begin{array}{l}\text { PedsQL-CSR } \\
\text { TSS } \\
\text { PHSS } \\
\text { PsHSS } \\
\text { Emotional } \\
\text { Social } \\
\text { School }\end{array}$ & $\begin{array}{l}71.41 \text { (II.26) } \\
75.31 \text { (16.43) } \\
69.33 \text { (9.63) } \\
75.00 \text { (19.29) } \\
69.50 \text { (16.57) } \\
63.50 \text { (I0.01) }\end{array}$ & $\begin{array}{l}81.85 \text { (I2.96) } \\
82.34 \text { (I5.72) } \\
81.58 \text { (I4.74) } \\
80.25 \text { (14.55) } \\
91.25 \text { (14.50) } \\
73.25(20.86)\end{array}$ & $\begin{array}{l}\mathbf{0 . 0 9 8} \\
0.305 \\
\mathbf{0 . 0 6 4} \\
0.579 \\
\mathbf{0 . 0 2 9} \\
0.287\end{array}$ \\
\hline $\begin{array}{l}\text { CBCL }^{\mathbf{a}} \\
\text { Anxious/depressed } \\
\text { Withdrawn/ } \\
\text { depressed } \\
\text { Somatic complaints } \\
\text { Social problems } \\
\text { Thought problems } \\
\text { Attention problems } \\
\text { Rule breaking } \\
\text { behaviour } \\
\text { Aggressive behaviour } \\
\text { Other problems } \\
\text { Total problems }\end{array}$ & $\begin{array}{l}4.40(2.80) \\
4.30(3.23) \\
4.10(2.69) \\
5.60(1.90) \\
4.00(2.40) \\
5.90(1.97) \\
2.40(1.26) \\
6.70(2.26) \\
4.20(2.25) \\
41.60(10.16)\end{array}$ & $\begin{array}{l}1.75(2.29) \\
2.85(2.68) \\
4.65(4.18) \\
2.30(2.15) \\
2.15(2.25) \\
3.20(3.05) \\
2.05(3.17) \\
3.85(3.73) \\
3.05(2.31) \\
25.85(19.04)\end{array}$ & $\begin{array}{l}0.029 \\
0.287 \\
0.983 \\
0.006 \\
0.102 \\
0.042 \\
0.287 \\
\\
0.029 \\
0.287 \\
0.029\end{array}$ \\
\hline $\begin{array}{l}\text { CPRS } \\
\text { Conduct problem } \\
\text { Learning problem } \\
\text { Psychosomatic } \\
\text { problem } \\
\text { Impulsive-hyperactive } \\
\text { Anxiety } \\
\text { Hyperactivity index }\end{array}$ & $\begin{array}{l}0.30(0.25) \\
0.88(0.36) \\
0.28(0.30) \\
0.28(0.22) \\
0.35(0.34) \\
0.46(0.18)\end{array}$ & $\begin{array}{l}0.25(0.27) \\
0.46(0.52) \\
0.37(0.42) \\
0.23(0.36) \\
0.25(0.21) \\
0.28(0.34)\end{array}$ & $\begin{array}{l}0.579 \\
\mathbf{0 . 0 8 2} \\
0.579 \\
\\
0.313 \\
0.579 \\
\mathbf{0 . 0 9 8}\end{array}$ \\
\hline
\end{tabular}

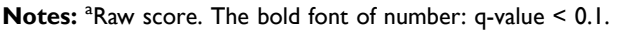

Abbreviations: CBCL, Child Behaviour Checklist; CPRS, Conners' Parent Rating Scales; CSR, child self-report; KPS, Karnofsky performance scales; LPS, Lansky playperformance scales; PedsQL, Pediatric Quality of Life Inventory 4.0 Generic Core Scales; PHSS, physical health summary score; PPR, parent proxy report; PsHSS, psychosocial health summary score; TSS, total scale score.

$50 \%$ of these patients had higher CBCL scores than the 95th percentile. In addition, the phenomenon that children with basal ganglia iGCTs showed various behavioral issues is consistent with the results reported by Park et al. ${ }^{18}$ 


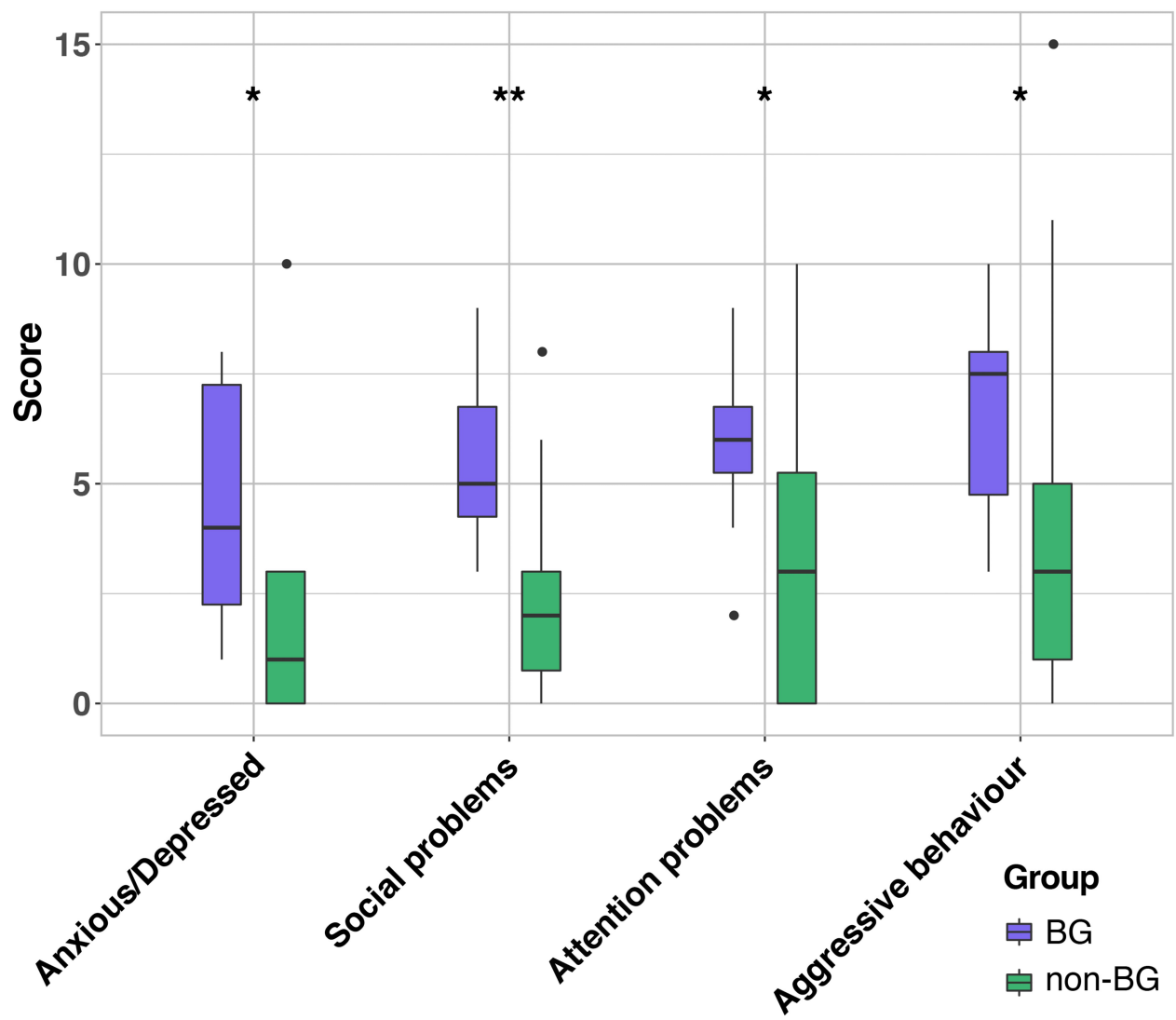

Figure I Main behavioral and emotional outcomes between the basal ganglia and the non-basal ganglia group. Patients with basal ganglia lesions (BG) had higher scores in the Child Behavior Checklist's anxious/depression, social problems, attention problems, and aggressive behavior sub-scores compared to those with non-basal ganglia lesions (non-BG). *q-value < 0.05; **q-value < 0.01; • outliers.

Our findings, to some extent, support the previous hypothesis that destruction of the specific basal ganglia network could directly trigger several behavioral and social problems. ${ }^{4-6}$

Patients with basal ganglia lesions had more attention problems and a higher hyperactivity index compared to those with non-basal ganglia lesions. The basal ganglia are closely linked to cognition and behavior through their dense and widespread projections to different cortical and limbic areas. Abnormal basal ganglia structure and function may lead to developmental disorders, ${ }^{6}$ poor cognitive performance, and difficulties in response control. ${ }^{19}$ Recent studies have shown that attention deficit is the basis for global cognitive decline, including declines in intellectual functioning and academic skills. Methylphenidate (MPH), which is the most commonly used drug for ADHD, enhances and maintains attention regulation and social skills among pediatric cancer survivors who have identified attention and learning problems. ${ }^{20}$ Over the course of one year, attention and behavioral benefits of MPH for childhood cancer survivors are maintained..$^{21}$ Early psychological interventions, such as using MPH, and the provision of more psychological support to children with basal ganglia iGCTs may improve their attention and behavior performance.

Imaging studies have shown that the activity of the putamen is related to repeatability and good learning. During new learning, the dorsolateral prefrontal cortex and striatum are activated. The frontal eye field sends projections to the striatum and body of the caudate nucleus, a circuit which also participates in learning. ${ }^{22}$ The basal ganglia are involved in multiple parallel isolation circuits with different regions of the frontal lobe, which is supported by anatomical evidence. ${ }^{23}$ This may explain why our patients with basal ganglia iGCTs had more learning problems compared to those with non-basal ganglia tumors.

Patients with basal ganglia lesions showed more aggressive behavior and we know that changes in basal ganglia regional volumes, metabolic function, and connectivity of specific neural networks are linked to 
aggression. $^{24}$ The basal ganglia play an important role in these circuits, such as participating in the neural reward circuits. When dealing with self-related undesirable information, activity increases in a network including the dorsomedial prefrontal cortex, hippocampus, thalamus, and ventral striatum. ${ }^{25}$ The imbalance of reward, fear, and cognitive control will generate aggressive behavior. The basal ganglia are also involved in the biology of aggression, such as the 5-HT system. The activity of 5-HT neurons is important for setting the threshold of aggressive behavior. There is a direct relationship between low levels of 5-HT metabolites and increased aggression. $^{24,26}$

In addition, more anxiety/depression was seen in patients with basal ganglia lesions than in those with nonbasal ganglia lesions. The basal ganglia are involved in the 5-HT system, which regulates almost all psychophysiological functions (such as anxiety, appetite, libido, reward, pain, memory, cognition, aggressiveness, response to stressors, and emotion). ${ }^{27}$ Future studies on 5-HT metabolites in the cerebrospinal fluid of patients with basal ganglia iGCTs may help to understand their aggressive behavior and emotional disorders.

Social, communication, and motor behaviors are connected to different areas of the basal ganglia through parallel projections from and to the frontal cortex. ${ }^{28}$ Social behavior and socio-emotional control are directly related to orbitofrontal and anterior cingulate loops with the medial caudate head. Basal ganglia impairments cause dyskinesia and social and communication disorders in autism spectrum disorder. ${ }^{29}$ Therefore, the invasion of iGCTs into the basal ganglia may damage the circuits, triggering social problems. The HRQoL of patients with basal ganglia lesions was worse than that of patients with non-basal ganglia lesions but there was no significant difference in PHSS of PedsQL and LPS/KPS scores. Social function impairment seems to be responsible for lower HRQoL in children with basal ganglia iGCTs.

Figure 1 shows that children with basal ganglia iGCTs have more behavioral and emotional problems than those with non-basal ganglia iGCTs. Although they did not affect the trend, we detected two outliers in the non-basal ganglia group. Interestingly, both patients had tumors in the pineal region and the pre-operative images showed the same feature: obvious bilateral edema of the basal ganglia due to tumor compression. The significantly higher scores of these two patients may be caused by this kind of damage to the basal ganglia, which highlights its importance in behavioral and emotional function.

Our study had some limitations. First, we analyzed a relatively small number of patients, ie only ten patients with basal ganglia iGCTs. Although recruiting patients with rare diseases is a huge challenge, the findings from the current study provide important evidence for the design of a larger and prospective study. We will examine more factors (eg, histological subtypes, family, and socioeconomic status) between groups to further validate the results. Second, the results do not represent the outcomes at the initial diagnosis before treatment since the study included children with a history of chemotherapy and surgery. The diagnosis of iGCTs is complex because some patients require biopsy and some patients need emergency surgery to relieve severe symptoms from hydrocephalus. There is no gold standard for the timing of surgical or chemotherapy intervention. Before the assessment, none of the patients received radiotherapy, which may be the most harmful treatment for cognitive function. Furthermore, we selected parent proxy scales to assess patients' behavior and asked them to match each item based on their child's current behavior and that in the past six months to reduce the impact of treatments on the outcomes. In addition, our T scores of the CBCL are based on the whole sample of the common Chinese population, so the influence of age and gender is not excluded. Finally, parent and self-rating scales were used to evaluate patients' outcomes. More objective evaluations, such as the Wechsler scale test and psychiatrist assessments, may be useful for better understanding this rare disease.

\section{Conclusion}

This study revealed that patients with basal ganglia iGCTs had more behavioral and emotional problems, social function impairment, and poorer HRQoL compared to patients with non-basal ganglia iGCTs. These findings may help to understand the function of the basal ganglia and provide evidence for the benefit of early psychological intervention to better treat this rare disease.

\section{Abbreviations}

iGCTs, intracranial germ cell tumours; HRQoL, healthrelated quality of life; LPS, Lansky play-performance scale; KPS, Karnofsky performance scale; PPR, parent proxy report; CSR, child self-report; CBCL, The Child Behaviour Checklist; CPRS, Conners' Parent Rating Scales; PedsQL, Pediatric Quality of Life Inventory; TSS, total scale score; PHSS, physical health summary score; PsHSS, psychosocial health summary score; M, mean; SD, standard deviation; ETV, endoscopic third ventriculostomy; V-P shunt, 
ventriculoperitoneal shunt; ADHD, attention deficit hyperactivity disorder; MPH, methylphenidate.

\section{Data Sharing Statement}

The datasets used and/or analyzed during the current study are available from the corresponding author on reasonable request.

\section{Ethics Approval and Informed Consent}

This study was approved by Beijing Tiantan Hospital. All participants provided informed consents.

\section{Consent for Publication}

Any details of this paper can be published.

\section{Acknowledgments}

This work was supported by the Capital Medical Development Research Fund (grant number 20202-1072). We are grateful to Dr. Chunxue Wang from the psychology department of Beijing Tiantan Hospital for supporting this study.

\section{Author Contributions}

All authors made substantial contributions to conception and design, acquisition of data, or analysis and interpretation of data; took part in drafting the article or revising it critically for important intellectual content; agreed on the journal to which the article will be submitted; gave final approval of the version to be published; and agree to be accountable for all aspects of the work.

\section{Funding}

This work was supported by The Capital Medical Development Research Fund (Grant Numbers: 20202-1072).

\section{Disclosure}

The authors declare that they have no competing interests.

\section{References}

1. McCarthy BJ, Shibui S, Kayama T, et al. Primary CNS germ cell tumors in Japan and the United States: an analysis of 4 tumor registries. Neuro-Oncology. 2012;14(9):1194-1200. doi:10.1093/ neuonc/nos 155

2. Zhang S, Liang G, Ju Y, You C. Clinical and radiologic features of pediatric basal ganglia germ cell tumors. World Neurosurg. 2016;95:516-524.e511. doi:10.1016/j.wneu.2016.08.072
3. Wang $\mathrm{M}$, Zhou $\mathrm{P}$, Zhang $\mathrm{S}$, et al. Clinical features, radiologic findings, and treatment of pediatric germ cell tumors involving the basal ganglia and thalamus: a retrospective series of 15 cases at a single center. Childs Nerv Syst. 2018;34(3):423-430. doi:10.1007/ s00381-017-3632-6

4. Graybiel AM. The basal ganglia: learning new tricks and loving it. Curr Opin Neurobiol. 2005;15(6):638-644. doi:10.1016/j.conb.2005.10.006

5. Bogacz R. Optimal decision-making theories: linking neurobiology with behaviour. Trends Cogn Sci. 2007;11(3):118-125. doi:10.1016/j. tics.2006.12.006

6. Sandman CA, Head K, Muftuler LT, Su L, Buss C, Davis EP. Shape of the basal ganglia in preadolescent children is associated with cognitive performance. NeuroImage. 2014;99:93-102. doi:10.1016/j. neuroimage.2014.05.020

7. Maia TV, Frank MJ. From reinforcement learning models to psychiatric and neurological disorders. Nat Neurosci. 2011;14(2):154-162. doi:10.1038/nn.2723

8. Liang SY, Yang TF, Chen YW, et al. Neuropsychological functions and quality of life in survived patients with intracranial germ cell tumors after treatment. Neuro Oncol. 2013;15(11):1543-1551. doi:10.1093/neuonc/not127

9. Rescorla LA, Achenbach TM. Manual for the ASEBA School-Age Forms and Profiles. Burlington, VT: University of Vermont Research Center for Children, Youth, and Families; 2001.

10. Cui Y, Li F, Leckman JF, et al. The prevalence of behavioral and emotional problems among Chinese school children and adolescents aged 6-16: a national survey. Eur Child Adolesc Psychiatry. 2020. doi:10.1007/s00787-020-01507-6

11. Shaw P, Gilliam M, Liverpool M, et al. Cortical development in typically developing children with symptoms of hyperactivity and impulsivity: support for a dimensional view of attention deficit hyperactivity disorder. Am $\quad J \quad$ Psychiatry. 2011;168(2):143-151. doi:10.1176/appi.ajp.2010.10030385

12. Goyette $\mathrm{CH}$, Conners $\mathrm{CK}$, Ulrich RF. Normative data on revised conners parent and teacher rating scales. J Abnorm Child Psychol. 1978;6(2):221-236. doi:10.1007/BF00919127

13. Varni JW, Seid M, Kurtin PS. PedsQL 4.0: reliability and validity of the pediatric quality of life inventory version 4.0 generic core scales in healthy and patient populations. Med Care. 2001;39(8):800-812. doi:10.1097/00005650-200108000-00006

14. Varni JW, Limbers CA. The PedsQL 4.0 generic core scales young adult version: feasibility, reliability and validity in a university student population. J Health Psychol. 2009;14(4):611-622. doi:10.1177/ 1359105309103580

15. Lansky LL, List MA, Lansky SB, Cohen ME, Sinks LF. Toward the development of a play performance scale for children (PPSC). Cancer. 1985;(56):1837-1840.

16. Roa W, Kepka L, Kumar N, et al. International atomic energy agency randomized Phase III Study of radiation therapy in elderly and/or frail patients with newly diagnosed glioblastoma multiforme. $J$ Clin Oncol. 2015;33(35):4145-4150. doi:10.1200/JCO.2015.62.6606

17. Reeve BB, McFatrich M, Mack JW, et al. Validity and reliability of the pediatric patient-reported outcomes - common terminology criteria for adverse events. $J$ Natl Cancer Inst. 2020;112(11):1143-1152. doi:10.1093/jnci/djaa016

18. Park Y, Yu ES, Ha B, Park HJ, Kim JH, Kim JY. Neurocognitive and psychological functioning of children with an intracranial germ cell tumor. Cancer Res Treat. 2017;49(4):960-969. doi:10.4143/crt.20 16.204

19. Qiu A, Crocetti D, Adler M, et al. Basal ganglia volume and shape in children with attention deficit hyperactivity disorder. Am J Psychiatry. 2009;166(1):74-82. doi:10.1176/appi.ajp.2008.08030426

20. Thompson SJ, Leigh L, Christensen R, et al. Immediate neurocognitive effects of methylphenidate on learning-impaired survivors of childhood cancer. J Clin Oncol. 2001;19(6):1802-1808. doi:10.1200/JCO.2001.19.6.1802 
21. Conklin HM, Reddick WE, Ashford J, et al. Long-term efficacy of methylphenidate in enhancing attention regulation, social skills, and academic abilities of childhood cancer survivors. J Clin Oncol. 2010;28(29):4465-4472. doi:10.1200/JCO.2010.28.4026

22. Yin HH, Mulcare SP, Hilário MR, et al. Dynamic reorganization of striatal circuits during the acquisition and consolidation of a skill. Nat Neurosci. 2009;12(3):333-341. doi:10.1038/nn.2261

23. Alexander GE, DeLong MR, Strick PL. Parallel organization of functionally segregated circuits linking basal ganglia and cortex. Anпи Rev Neurosci. 1986;9(1):357-381. doi:10.1146/annurev.ne.09. 030186.002041

24. Cupaioli FA, Zucca FA, Caporale C, Lesch KP, Passamonti L, Zecca L. The neurobiology of human aggressive behavior: neuroimaging, genetic, and neurochemical aspects. Prog Neuropsychopharmacol Biol Psychiatry. 2021;106:110059. doi:10.1016/j.pnpbp.2020.110059.

25. Kuzmanovic B, Jefferson A, Vogeley K. The role of the neural reward circuitry in self-referential optimistic belief updates. NeuroImage. 2016;133:151-162. doi:10.1016/j.neuroimage.2016. 02.014
26. Audero E, Mlinar B, Baccini G, Skachokova ZK, Corradetti R, Gross C. Suppression of serotonin neuron firing increases aggression in mice. $J$ Neurosci. 2013;33(20):8678-8688. doi:10.1523/JNEUROSCI.206712.2013

27. Charnay Y, Léger L. Brain serotonergic circuitries. Dialogues Clin Neurosci. 2010;12(4):471-487.

28. Kumfor F, Sapey-Triomphe LA, Leyton CE, Burrell JR, Hodges JR, Piguet O. Degradation of emotion processing ability in corticobasal syndrome and Alzheimer's disease. Brain. 2014;137(11):3061-3072. doi:10.1093/brain/awu246

29. Qiu A, Adler M, Crocetti D, Miller MI, Mostofsky SH. Basal ganglia shapes predict social, communication, and motor dysfunctions in boys with autism spectrum disorder. $J$ Am Acad Child Adolesc Psychiatry. 2010;49(6):534-539. doi:10.1016/j.jaac.2010.02.012

\section{Publish your work in this journal}

Neuropsychiatric Disease and Treatment is an international, peerreviewed journal of clinical therapeutics and pharmacology focusing on concise rapid reporting of clinical or pre-clinical studies on a range of neuropsychiatric and neurological disorders. This journal is indexed on PubMed Central, the 'PsycINFO' database and CAS, and is the official journal of The International Neuropsychiatric Association (INA). The manuscript management system is completely online and includes a very quick and fair peer-review system, which is all easy to use. Visit http://www.dovepress.com/testimonials.php to read real quotes from published authors. 